\title{
Relaciones entre estrés académico, apoyo social, optimismo-pesimismo y autoestima en estudiantes universitarios
}

\section{Fernández González, L. ${ }^{1}$, González Hernández, A. ${ }^{2}$ y Trianes Torres, M.V. ${ }^{3}$}

\author{
${ }^{1}$ Doctorado en Psicología. Universidad de Málaga. \\ ${ }^{2}$ Departamento de Psicología Social, Antropología Social, Trabajo Social y \\ Servicios Sociales, Universidad de Málaga. \\ ${ }^{3}$ Departamento de Psicología Evolutiva y de la Educación, Universidad de \\ Málaga.
}

\section{España}

Correspondencia: $\mathrm{M}^{\mathrm{a}}$ Victoria Trianes. Facultad de Psicología. Campus de Teatinos. Universidad de Málaga, CP: 29071. Málaga. España E-mail: triatorr@uma.es 


\section{Resumen}

Introducción. El objetivo de la presente investigación fue analizar de la manera en la cual variables como el optimismo, el pesimismo, la autoestima y el apoyo social tienen un efecto sobre el estrés académico en estudiantes universitarios.

Método. La muestra estuvo constituida por 123 estudiantes de $3^{\circ}$ de Psicología con edades comprendidas entre los 20 y 31 años. Los estudiantes cumplimentaron la escala de autoestima de Rosenberg, el cuestionario de optimismo Life Orientation Test (LOT-R), el cuestionario de frecuencia y satisfacción con el apoyo social y el Student Stress Inventory, Stress Manifestation (SSI-SM). Se realizaron tres análisis de regresión lineal, en cada análisis se empleó una variable dependiente distinta: manifestaciones de estrés fisiológicas, conductuales o emocionales; y como variables predictoras: optimismo, pesimismo, autoestima y satisfacción con el apoyo social (emocional, instrumental e informacional).

Resultados. Los resultados de cada uno de los análisis muestran que, en primer lugar, las manifestaciones fisiológicas del estrés no son predichas por las variables predictoras utilizadas, en segundo lugar, las manifestaciones conductuales de estrés han obtenido resultados significativos en relación con la satisfacción con el apoyo social instrumental y el optimismo. En tercer lugar, tomando las manifestaciones emocionales de estrés como variable dependiente, se observan resultados significativos respecto al optimismo y el pesimismo. En cuanto a la autoestima no ha resultado ser significativa. Finalmente se ha realizado un MANOVA que muestra efectos principales del optimismo, la satisfacción con el apoyo instrumental, la edad y el sexo.

Discusión. Las hipótesis previstas en el estudio se han cumplido parcialmente.

Palabras clave: Autoestima, optimismo, apoyo social, estrés académico y estudiantes universitarios

Recibido: 09/11/14 Aceptación Inicial: 12/01/15 Aceptación final: 14/03/15 


\title{
Relationships between academic stress, social support, optimism-pessimism and self-esteem in college students
}

\begin{abstract}
Introduction. This research aims to analyse how optimism, self-esteem and social support help to predict academic stress.

Method. The sample consisted of 123 students aged 20 to 31 years old, from the 3rd Year in the Psychology Degree.. Students completed the Rosenberg Self-esteem Scale, the Life Orientation Optimism Questionnaire (LOT-R), the Frequency and Satisfaction with Social Support Questionnaire and the Student Stress Inventory - Stress Manifestation (SSI-SM). Three linear regression analyses were conducted, each on a different variable: manifestations of physiological, behavioral and emotional stress. The predictor variables were: optimism, pessimism, self-esteem and frequency and satisfaction with social (emotional, instrumental and informational) support.
\end{abstract}

Results. Results showed that the physiological manifestations of stress are not predicted by the used predictor variables, while there is significant proof that the behavioral manifestations of stress are predicted by satisfaction with instrumental social support and optimism. There is significant proof that the emotional manifestations of stress are predicted by optimism and pessimism. As for self-esteem, it did not prove to be significant. Finally, a MANOVA analysis was conducted, showing the main effects to be optimism, satisfaction with instrumental support, age and gender.

Discussion. The hypotheses of the study have been partially met.

Key words: Self-esteem, optimism, social support, academic stress and university students.

Reception: 11/01/14 Initial acceptance: 01/11/15_ Final acceptance: 03/14/15 


\section{Introducción}

Hoy se tiene conciencia de que la sociedad vive con estrés muchas de sus situaciones de vida. El estrés está presente tanto en la vida de las personas como se argumenta como causas de psicopatología, como causas de enfermedad, y como causas de inadaptación al ambiente o al trabajo (Feldman, Goncalves, Chacón-Puignau, Zaragoza, Bagés, y de Pablo, 2008). Quizás sea la población universitaria una de las menos estudiadas en cuanto al estrés (Martín 2007). Sin embargo puede asumirse que los estudiantes en la universidad sufren estrés que se puede calificar como académico (De la Fuente et al, 2014). Este estrés académico puede guardar relación con los exámenes y frecuentes evaluaciones, con la presión de los trabajos y tareas diarias, con las dificultades económicas que impactan en la calidad del estudio, con dificultades familiares o de otra índole que puede sufrir el estudiante dificultándole alcanzar el éxito (; Martín, 2007). También pueden los estudiantes manifestar otros tipos de estrés como emocional, psicofisiológico, o conductual (Maldonado, Trianes, Cortés, Moreno y Escobar, 2009; Muñoz, 1999).

El estrés se considera un constructo complejo que se produce en dos fases, en la primera la persona valora cognitivamente el estresor y las demandas que este le suscita y en un segundo lugar evalúa si posee recursos suficientes para afrontar las demandas del estresor (Lazarus y Folkman, 1986). Este punto de vista pone la atención en la valoración subjetiva del estresor que determina la reacción ante él. Los acontecimientos que producen estrés son llamados estresores (Holmes y Rahe, 1967). Personas diferentes pueden reaccionar de modo diferente ante un mismo estresor (Arribas, 2013). El estrés como respuesta hace referencia a las reacciones del organismo, ya sean de tipo fisiológico (Selye, 1978), emocional o comportamental ante estímulos que provocan estrés (Blanca, Fernández-Baena, y Trianes, 2011; Fimian, Fastenau, Tashner y Cross, 1989). El enfoque actual sobre el estrés lo considera un resultante de la reacción de la persona con el entorno (Escobar, Blanca, Fernández-Baena, y Trianes, 2011). Este enfoque integra las consideraciones del estrés como estímulo y como respuesta. En definitiva el estrés surge cuando la persona evalúa un acontecimiento del entorno y cree que es amenazante y que pone en peligro su bienestar, ya que no cuenta con los recursos suficientes para enfrentarse a dichos aspectos amenazantes (Lazarus y Folkman, 1986). 


\section{Estrés Académico}

Se habla de estrés académico en el caso de que los estresores y respuestas pertenezcan al mundo educativo. En una investigación (Feldman y cols, 2008), se ha observado que la intensidad del estrés académico disminuye a medida que se incrementa el apoyo social. En otros estudios los investigadores han encontrado que los estudiantes universitarios experimentan altos niveles de estrés, sobre todo cuando comienzan una carrera y en las épocas previas a los exámenes (Muñoz, 1999) mientras que el estrés disminuye en los últimos cursos. Por otra parte, se ha considerado que los estresores académicos fundamentales pueden ser las notas finales, demasiado trabajo para casa, los exámenes y estudiar para superarlos (Kohn y Frazer, 1986; Misra y McKean, 2000).

Hay trabajos que muestran problemas de salud producidos por la experiencia de sufrir estrés académico (Fernández, Siegrist, Rödel y Hernández, 2003). Hernández, Pozo y Polo (1994) investigaron de qué manera afectan los cambios que se producen en la vida de los estudiantes a medida que se aproxima el período de exámenes, encontrando la aparición de hábitos perjudiciales para la salud (consumo excesivo de cafeína, tabaco y sustancias psicoactivas). Otros investigadores (Smith y Ellsworth (1987, citado por Muñoz, 2003) verificaron que los esquemas emocionales y la valoración de la realidad se modificaba principalmente desde el periodo previo a los exámenes hasta una vez proporcionada la información de las notas.

El estrés también se acusa a nivel psicofisiológico, habiéndose detectado que existen hormonas ligadas a la experiencia del estrés como Cortisol y también otras reacciones del Sistema Nervioso parasimpático se asocian a la experiencia del estrés (Maldonado, Trianes, Cortes, Moreno, y Escobar, 2009). Hay trabajos que muestran problemas de salud producidos por el estrés académico como el de Kiecolt, Glaser, Strian, Stout, Tarr, Holliday y Speicher, (1986), quienes realizaron un estudio con estudiantes de medicina resultando que el funcionamiento de algunas células del sistema inmunitario se eliminan durante la época de exámenes lo que hace que el organismo sea más propenso a sufrir enfermedades.

\section{Autoestima}


Dado que la autoestima influye también en los resultados académicos se ha seleccionado para formar parte del patrón relevante que se asume en la presente investigación que puede explicar calidad de vida en universitarios. Se define esta variable, según Rosenberg (1965), como la actitud integral que tiene la persona hacia sí misma, ya sea positiva o negativa. En el terreno académico la autoestima es una variable relevante ya que se asocia a buenos resultados escolares y a motivación intrínseca hacia el aprendizaje (Navarro, Tomás y Oliver 2006). Así mismo baja autoestima se asocia a rechazo de los iguales (Murray, Griffin, Rose y Bellavia, 2003) psicopatología internalizada (González-Fortaleza y Ruiz,1993), así como fracaso escolar y abandono de los estudios (Navarro y cols, 2006). En estudiantes universitarios existe relación entre autoestima y bienestar psicológico (Diener y Diener, 1995; Paradise y Kernis, 2002; Kang, Shaver, Sue, Min y Jing, 2003; Reid, 2004). Incluso se ha encontrado que la autoestima influye en el bienestar psicológico en mayor medida que el apoyo social percibido (Barra , 2012).

Uno de los instrumentos más conocidos para la evaluación de la autoestima ha sido la Escala de Autoestima de Rosenberg (EAR) (Rosenberg, 1965; Schmitt y Allik, 2005) que fue planteada por su autor como medida unidimensional, coincidiendo con otros estudios ((Martín, Núñez, Navarro, y Grijalvo, 2007). Por el contrario, se ha comprobado también su multidimensionalidad (Manso, 2010) concluyendo que depende de la población dónde se aplique, así como del sentido en que se enuncian algunos de sus ítems, ya sean en positivo o negativo (Goldsmith, 1986).

\section{Optimismo / Pesimismo}

También el optimismo/ pesimismo han sido seleccionados en la presente investigación formando parte del mismo conjunto de variables relevantes para el bienestar psicológico en universitarios. El optimismo se puede definir como la predisposición que posee el individuo hacia la creencia de que los resultados que desea los puede obtener sin grandes dificultades. El optimismo forma parte de una dimensión en cuyo otro extremo está el pesimismo (Ferrando, Chico y Tous, 2002). Pesimismo se define al contrario que el optimismo como creencias en que se va a obtener resultados negativos o dificultades añadidas (Londoño, 2009). El optimismo podría desempeñar un papel importante a la hora de utilizar conductas de afrontamiento adaptativas (Scheier y Carver,1985), influyendo en el bienestar psicológico y físico (Chang, D’Zurilla y Maydeu-Olivares, 1994), desempeñando un importante papel amortiguador de riesgos de enfermedades físicas y psicológicas (Peterson, Seligman y 
Vaillant, 1988), además de relacionarse con resultados positivos sobre el estado de salud físico y psicológico de las personas (Remor, Amorós y Carboles, 2006). En estudiantes universitarios se encontró que el optimismo determina la permanencia del alumnado, predice la repetición y desactiva el riesgo de abandono de las materias (Londoño, 2009).

Incluso es concebido el optimismo como un rasgo disposicional de la personalidad que actúa como mediador en hechos que acontecen de manera externa al sujeto y en la interpretación individual de éstos (Seligman, 1990). Además, este autor (2003) plantea que este constructo se puede explicar como predisposición del individuo a atribuir los acontecimientos negativos a causas externas al sujeto, inestables en el tiempo y en situaciones concretas de la vida. Por el contrario, el pesimismo se puede entender como una predisposición a explicar que los acontecimientos negativos suceden por causas internas a las personas, que se mantienen estables en el tiempo y pueden ser extendidos a diversas áreas de la vida. Se pueden considerar optimismo y pesimismo como perspectivas sistematizadas (positivas y negativas) sobre los sucesos que nos ocurren en la vida (Scheier y Carver, 1985) por lo que algunos lo denominan optimismo/pesimismo disposicional.

\section{Apoyo Social}

Otra de las variables utilizadas en la presente investigación ha sido el apoyo social. Este es uno de los factores de protección más investigado y con resultados más favorables para incrementar la resistencia de las personas ante los cambios vitales, situaciones estresantes, crisis personales y afrontamiento de enfermedades, sobre todo las crónicas (Feldman y cols,2008). Puede definirse el apoyo social como la ayuda real y percibida, de la comunidad, las redes sociales y amigos cercanos ya sea de tipo instrumental o emocional (Hombrados, Gómez, Domínguez, García y Castro, 2012). Se llama apoyo instrumental a la emisión de conductas que directamente ayudan a quien lo necesita, por ejemplo cuidar a alguien mientras que se llama apoyo emocional a variables de cuidado, amor, confianza, (Hombrados, García y Gómez, 2013). En el presente trabajo se evalúa el apoyo social informacional, instrumental y emocional.

Haciendo referencia al ámbito académico, el apoyo social puede facilitarse a través de aquellas personas cercanas a los estudiantes y de aquellos recursos disponibles, lo que hace que proporcione una ayuda emocional segura, que los estudiantes tengan oportunidades para compartir sus intereses y que se sientan comprendidos y respetados (Feldman y cols, 2008). 
Diversos autores han encontrado que este constructo podría fomentar que los estudiantes obtengan unos resultados académicos satisfactorios (Román y Hernández, 2005) y, además, que el apoyo social ayuda a los estudiantes a enfrentarse a los estresores con probabilidad de éxito (Martín, 2007).

\section{Objetivos e hipótesis}

La presente investigación tiene como objetivo general analizar de qué forma interaccionan unas variables que por separado han demostrado guardar relación con calidad de vida en universitarios. En concreto deseamos probar si el estrés académico puede predecirse a partir del apoyo social más autoestima, optimismo y pesimismo en universitarios. Se espera que optimismo, pesimismo, apoyo social y autoestima predigan de forma significativa el estrés académico de los estudiantes.

\section{Método}

\section{Participantes}

Participan en este estudio 118 estudiantes de la Universidad de Málaga (España) (93 mujeres y 25 hombres), con edades comprendidas entre los 20 y los 31 años (Media= 21.4 años, $\mathrm{SD}=0.41$ ). Eran estudiantes de $3^{\circ}$ de Psicología de la Universidad de Málaga (España). Los datos se tomaron en el curso académico 2012-13. La voluntariedad se pidió en clase verbalmente y ningún alumno rehusó participar. Como son futuros psicólogos tienen asumido el participar en investigaciones.

\section{Instrumentos}

Autoestima. Para medir la autoestima, hemos utilizado la Escala de Autoestima de Rosenberg (EAR) (Rosenberg, 1965; Schmitt y Allik, 2005). Hemos utilizado la escala original traducida en una agencia de traducción (www.t-esperanto.com). Nos indican los revisores que hay una adaptación al castellano (Baños y Guillen, 2000). Esta escala está compuesta por 10 ítems con formato de respuesta tipo Likert con 4 opciones de respuesta, que van desde muy en desacuerdo (1) a muy de acuerdo (4). Para reducir el efecto aquiescencia, cinco de los ítems fueron construidos positivamente y cinco redactados negativamente. Debido a que las puntuaciones altas y bajas obtuvieron el mismo resultado, las puntuaciones obtenidas en los ítems negativos fueron transformadas a ítems positivos. Así, la puntuación 
total obtenida en la escala oscila en un rango de 10 a 40, dónde la puntuación 10 indica autoestima baja y la puntuación 40 autoestima alta. Este cuestionario ha demostrado tener un coeficiente Alpha de Cronbach apropiado (Alpha= 0.83). Los autores de este artículo señalan que en la adaptación española de Baños y Guillén (2000) el coeficiente alpha es de 0.88.

Optimismo. El optimismo se ha evaluado a través de la adaptación española (Ferrando, Chico y Tous, 2002) de la versión revisada del Life Orientation Test (LOT-R) (Scheier, Carver y Bridges, 1994). El test consta de 10 ítems con formato de respuesta tipo Likert de 5 puntos. De éstos, 6 de ellos tratan medir la dimensión de optimismo disposicional, mientras que los 4 restantes sirven para que sea menos evidente el contenido del test. De los 6 ítems comentados anteriormente, 3 se presentan en sentido positivo (dirección optimismo) y 3 en sentido negativo (dirección pesimismo). Los ítems que están redactados de forma negativa se invierten y así, se puede obtener una puntuación total orientada hacia el polo de optimismo. Dichas puntuaciones van desde totalmente en desacuerdo (1) a de acuerdo (5). Los autores reportan índices de fiabilidad y validez aceptables.

Apoyo social. Para valorar el apoyo social se ha empleado el Cuestionario de Frecuencia y Satisfacción con el Apoyo Social (Hombrados, et al, 2012). Mide la frecuencia de apoyo y el nivel de satisfacción con el mismo. En él, se pueden analizar las distintas fuentes de apoyo (padre, madre, compañeros de clase y profesores) y el tipo de apoyo (emocional, instrumental e informacional). Además, dicho cuestionario, valora la frecuencia de apoyo recibido de la red social y el grado de satisfacción con el mismo. Se trata de una escala tipo Likert con 5 opciones de respuesta tanto en la frecuencia de apoyo recibido como en el grado de satisfacción, que van desde nunca (1) hasta siempre (5) (para la frecuencia) y desde insatisfecho (1) hasta muy satisfecho (5) (para la satisfacción). El coeficiente Alpha de Cronbach hallado en este cuestionario es de 0.91. Además, en este instrumento, también se han hallado los coeficientes Alpha de los diferentes tipos de apoyo. En el apoyo emocional, Alpha $=0.780$, en el apoyo instrumental Alpha= 0.763 y en el apoyo informacional Alpha= 0.795. En cuanto a la frecuencia y satisfacción los coeficientes Alpha de Cronbach son: para la frecuencia Alpha $=0.840$ y para la satisfacción Alpha $=0.867$.

Estrés. La evaluación del estrés académico se ha realizado utilizando la adaptación española (Escobar, Blanca, Fernández-Baena y Trianes, 2011) de la Escala de Manifestaciones de Estrés del Student Stress Inventory - Stress Manifestations (SSI-SM) (Fimian et al., 1989). Está constituida por 22 ítems con un formato de respuesta tipo Likert de 
5 opciones que oscilan entre (1) en absoluto hasta (5) totalmente, que engloban tres ámbitos de manifestaciones de estrés: emocionales (10 ítems), fisiológicas (6 ítems) y conductuales (6 ítems). Los valores del coeficiente alfa de consistencia interna calculados son de 0,79 para el factor de manifestaciones emocionales, 0,62 para manifestaciones fisiológicas y 0,66 para manifestaciones conductuales. El valor más alto corresponde a la puntuación total del test, 0,86 .

\section{Procedimiento}

Para llevar a cabo esta investigación, lo primero que se hizo, fue contactar con los profesores/as que imparten las asignaturas "Dificultades en el Aprendizaje Escolar" y "Psicología Comunitaria” en el Grado en Psicología en la Universidad de Málaga (España), para poder asistir a sus clases a administrar los cuestionarios. Una vez concedida la autorización, los test se aplicaron durante una mañana para los grupos A y B en Psicología Comunitaria y dos tardes para los grupos C y D en Dificultades en el Aprendizaje Escolar. A todos los grupos, antes de repartir los instrumentos, se les explicó brevemente el motivo por el que se realizaba esta investigación, cómo debían cumplimentar los cuestionarios y se pedía la voluntariedad de su participación en la misma clase. El tiempo que emplearon para cumplimentar todos los instrumentos sin dificultades, fue de aproximadamente, unos 20 minutos.

\section{Análisis de datos y diseño}

Se realizaron tres análisis de regresión lineal (método de introducción: pasos sucesivos), en cada análisis se empleó una variable dependiente distinta: manifestaciones de estrés fisiológicas, conductuales o emocionales; y como variables predictoras, optimismo, pesimismo, autoestima y satisfacción con el apoyo social (emocional, instrumental e informacional).

De manera complementaria hemos realizado un análisis multivariante, en el cual se han introducido, como variables dependientes, de nuevo las manifestaciones de estrés (emocionales, fisiológicas y conductuales), Satisfacción con el apoyo Emocional, Satisfacción con el apoyo Instrumental y Satisfacción con el apoyo Informacional. Como covariables, el sexo, la edad, optimismo, pesimismo, autoestima, así como la frecuencia y satisfacción del apoyo emocional, instrumental e informacional. 


\section{Resultados}

\section{Resultados predictivos}

Análisis de regresión 1. Si introducimos como variable dependiente el estrés fisiológico, los resultados nos ofrecen un modelo no significativo $(p>, 05)$. Las manifestaciones fisiológicas del estrés no son predichas por las variables independientes empleadas en el estudio.

Análisis de regresión 2. Si introducimos como variable dependiente el estrés conductual, obtenemos dos modelos significativos: el primero incluyendo sólo la Satisfacción con el Apoyo Social Informacional como variable predictora, $F(1,116)=12,762, p<.001 ; \mathrm{y}$ el segundo incluyendo tanto la Satisfacción con el Apoyo Social Instrumental como el Optimismo $F(2,115)=10,012, p<.000$. Ver tabla 1 para coeficientes.

Tabla 1. Coeficientes análisis de regresión 2

\begin{tabular}{|c|c|c|c|c|c|c|}
\hline Modelo & & Coeficier & ates no estand. & Coeficientes stand. & $\mathrm{t}$ & Sig. \\
\hline & & B & Error típ. & Beta & B & Error típ. \\
\hline \multirow[t]{2}{*}{1} & (Constante) & 15,606 & 1,547 & & 10,088 & 000 \\
\hline & SAT.AP.INFORM & $4 \quad-, 348$ & ,098 &,- 315 & $-3,572$ &, 001 \\
\hline \multirow[t]{3}{*}{2} & (Constante) & 18,174 & 1,810 & & 10,043 &, 000 \\
\hline & SAT.AP.INFORM & I --297 & 097 &,- 269 & $-3,058$ & 8,003 \\
\hline & OPTIMISMO &,- 313 &, 122 &,- 227 & $-2,577$ & 7,011 \\
\hline
\end{tabular}

a Variable dependiente: Estrés Conductual

Análisis de regresión 3. Por último, usando el estrés emocional como variable dependiente, de nuevo encontramos resultados significativos en dos modelos: el primero incluyendo sólo el optimismo como variable predictora, $F(1,116)=34,423, p<.001$; y el segundo incluyendo tanto optimismo como pesimismo $F(2,115)=19,726, p<.001$. Ver tabla 2 para coeficientes). 
Tabla 2. Coeficientes análisis de regresión 3

\begin{tabular}{|c|c|c|c|c|c|c|}
\hline Modelo & & Coeficien & ntes no estand. & Coeficientes stand. & $\mathrm{t}$ & Sig. \\
\hline & & B & Error típ. & Beta & B & Error típ. \\
\hline \multirow[t]{2}{*}{1} & (Constante) & 40,620 & 2,875 & & 14,129 &, 000 \\
\hline & OPTIMISMO & $-1,540$ & 262 &,- 478 & $-5,867$ & 7,000 \\
\hline \multirow[t]{3}{*}{2} & (Constante) & 33,073 & 4,682 & & 7,064 &,+ 000 \\
\hline & OPTIMISMO & $-1,222$ & ,303 &,- 380 & $-4,033$ & 3,000 \\
\hline & PESIMISMO & !615 & ,304 & ,191 & 2,026 & 6,045 \\
\hline
\end{tabular}

a Variable dependiente: Estrés Emocional

\section{Resultados inferenciales}

Los resultados muestran un efecto marginalmente significativo del sexo, $F(6,107)=$ $2,081, p=.061 ; \mathrm{y}$ efectos significativos de la edad $\mathrm{F}(6,107)=2,352$ y el optimismo $F$ $(6,107)=3,541, p<.05$.

El sexo tiene un efecto sobre las manifestaciones emocionales (las mujeres muestran más manifestaciones emocionales que los hombres) y fisiológicas del estrés (las mujeres muestran más manifestaciones fisiológicas que los hombres. El efecto principal de la edad se manifiesta sobre la satisfacción con el apoyo instrumental (gráfico 3). En general, conforme se incrementa la edad de los participantes, la satisfacción que tienen con el apoyo instrumental disminuye.

El optimismo tiene efecto sobre las manifestaciones emocionales, en general conforme se incrementa el optimismo, menos manifestaciones emocionales ocurren. También hay un efecto sobre las manifestaciones conductuales; en general, a mayor optimismo, menos manifestaciones conductuales de estrés aparecen.

\section{Discusión y conclusiones}

Las hipótesis previstas se han cumplido parcialmente. De las variables implicadas, la autoestima no ha mostrado resultados significativos, quizás por ser una variable interna formada por evaluaciones a medio y largo plazo (Barra, 2012) quizás poco sujeta a cambios 
situacionales y poco permeable al impacto de estresores diversos que parecen tener mayores consecuencias en el ámbito emocional.

El primer análisis de regresión sobre el estrés fisiológico no muestra resultados significativos. Quizás porque la índole de las variables utilizadas en este estudio se alejan de este ámbito psicofisiológico aunque el mundo emocional si tiene relaciones con variables fisiológicas. En el presente artículo cuando se divide la muestra por sexo si se obtiene un resultado de manifestaciones fisiológicas asociado a las mujeres que se comenta más adelante.

En el segundo análisis de regresión se obtiene que el estrés conductual es predicho por Satisfacción con el Apoyo Social Instrumental y el Optimismo. El apoyo social instrumental tiene que ver con recibir ayudas ante las dificultades lo que es efectivo para manejar el estrés. El optimismo permite ver los aspectos positivos de la vida. El estrés conductual se refiere a las conductas que debe ejecutar el sujeto para hacer frente a las demandas del estresor. El afrontamiento de las demandas del estresor se ve facilitado por poseer un temperamento optimista o bien por tener ayudas que permiten manejar mejor la ansiedad o las demandas del estresor. Son variables que van en el mismo sentido por lo que se relacionan entre sí. Otras investigaciones muestran también que el estrés por satisfacer las demandas del estresor se ve facilitado por disfrutar de ayudas relevantes (Polo, Hernández y Pozo, 1996) y por el optimismo (Martínez, Reyes del Paso, García y González, 2006).

En nuestros datos del tercer análisis de regresión efectuado, el estrés emocional es predicho por dos variables de índole emocional en parte, el optimismo y el pesimismo. El optimismo es visto hoy casi como una variable de personalidad (Ferrando, Chico y Tous, 2002) por lo que una persona optimista presenta protección eficaz frente al estrés, y la enfermedad física y psicológica (Peterson, Seligman y Vaillant, 1988). Se deriva de aquí la relevancia de educar en el optimismo preferentemente a jóvenes con pesimismo temperamental. En la universidad podría acometerse esta educación en titulaciones propias. También cabe destacar aquí que el hecho de que el apoyo emocional sea predicho por optimismo y pesimismo puede aludir a la relevancia del mundo emocional. En el análisis MANOVA el optimismo presenta además en nuestros datos un efecto reductor de las manifestaciones emocionales pues a medida que se incrementa disminuyen las manifestaciones emocionales del estrés. Hoy se reconoce la importancia del mundo emocional, tanto a nivel de inteligencia emocional (Shapiro, 1997; Goleman, 1996) como en 
cuanto a competencia emocional (Saarni, 1999) por lo que no es de extrañar que estos resultados se polaricen en torno a lo emocional.

De los resultados del MANOVA comentaremos ahora el asociado a la edad ya que, a medida que son mayores los estudiantes disminuye su satisfacción con el apoyo instrumental. Puede ser debido a que la edad proporciona más seguridad en sí mismo y por tanto puede actuar la persona con más autonomía sin depender del apoyo instrumental (Musitu y Cava, 2003).

Otros resultados se refieren al sexo encontrándose que las mujeres puntúan más alto tanto en manifestaciones emocionales del estrés como en manifestaciones fisiológicas. Este resultado coincide con otros trabajos atribuyéndose a la socialización de la mujer, a diferencia del hombre, más permisividad en las manifestaciones emocionales y más vulnerabilidad a sentimientos que le apartan de una respuesta decidida al estrés. En cuanto a puntuar más en manifestaciones fisiológicas se puede atribuir a la diferente fisiología de la mujer que acarrea mayor vulnerabilidad también para las vivencias de las enfermedades y síntomas del estrés (Escobar, Blanca, Fernández-Baena y Trianes, 2011). De hecho en otros trabajos las mujeres muestran más nivel de estrés y sintomatología emocional en profesionales de la salud de niveles primario y secundario (Roman, 2003) también en profesionales de enseñanza (Matud, García y Matud, 2002). En adolescentes también las mujeres reportan más estrés percibido y más ánimo depresivo (Barra, 2012). En universitarios igualmente el mayor nivel de estrés de las mujeres fue relacionado con pérdida de apoyo social de amigos (Feldman et al, 2008).

De este resultado se deriva la importancia de proporcionarse una educación emocional a niños y adolescentes así como en la universidad. Hoy existe un interés por cursos sobre inteligencia o competencias emocionales en la universidad. En la Facultad de Psicología de la Universidad de Málaga se ofertan cursos de Inteligencia Emocional para universitarios que se llenan cada año. También hay asignaturas de Inteligencia Emocional en posgrados de la UMA. Puede sugerirse que esta iniciativa se extienda a la secundaria obligatoria así como el bachillerato, un momento vital en el que se sufre particularmente estrés académico (Escobar et al, 2011).

También ofrece interés la educación en el optimismo, que es reconocido hoy como una variable central en la salud física y psíquica por sus efectos positivos sobre la adaptación al medio ambiente y por ofrecer un escudo frente a las tensiones y frustraciones relacionadas con la adversidad y los problemas de la vida. 
Una limitación de este trabajo es el no haber podido pasar más pruebas de emocionalidad y calidad de vida. Por tanto este trabajo podría mejorarse incluyendo otras variables de índole emocional, como autorregulación emocional, satisfacción vital, etc. mejor que variables internas como autoestima que no ofrece resultados.

\section{Agradecimientos}

Los autores agradecen a los revisores sus sugerencias para mejorar la calidad de este artículo. 


\section{Referencias}

Arribas, J. (2013). Hacia un modelo causal de las dimensiones del estrés académico en estudiantes de enfermería. Revista de Educación, 360,533-556. DOI: 10.4438/1988592X-RE-2011-360-126.

Baños, R.M. y Guillén, V. (2000). Psychometric characteristics in normal and social phobic samples for a Spanish version of Rosenberg Self Esteem Scale. Psychological Reports, 87, 269-274. DOI: 10.2466/PR0.87.5.269-274.

Barra, E. (2012). Influencia de la autoestima y del apoyo social percibido sobre el bienestar psicológico de estudiantes universitarios chilenos. Diversitas: Perspectivas en Psicología [edición online], 8 (1), 29-38.

Bérnia, D.L. y Barraza, A.(2005). El estrés académico de los alumnos de Educación Media Superior, Hermosillo. Memoria electrónica del VIII Congreso Nacional de Investigación Educativa.

Cabanach, R.G., Fariña, F., Freire, C., González, P.y Ferradás, M.M. (2013). Diferencias en el afrontamiento del estrés en estudiantes universitarios hombres y mujeres. European Journal of Education and Psychology, 6 (1), 19-32. DOI: 10.1989/ejep.v6i1.100.

Chang, E.C., D'Zurilla, T.J. y Maydeu-Olivares, A. (1994). Assessing the dimensionality of optimism and pessimism using a multimeasure approach. Cognitive Therapy and Research, 18(2), 143-160. DOI 10.1007/BF02357221.

De la Fuente, J., López, M., Zapata, L. Martínez-Vicente, J.M., Vera, M.M., Solinas, G., \& Fadda, S. (2014). Competency to study and learn in stressful contexts: fundamentals of the "e-Coping with Academic Stress" utility. Electronic Journal of Research in Educational Psychology 12(3), 717-746. DOI: http://dx.doi.org/10.14204/ejrep.34.14034

De Pablo, J., Baillés, E., Pérez, J. y Valdés, M. (2002). Construcción de una escala de estrés académico para estudiantes universitarios. Educación Médica, 5(1), 40-46.

Diener, E., y Diener, M. (1995). Cross-cultural correlates of life satisfaction and Self-Steem. Journal of Personality and Social Psychology, 68(4), 653-663.

Dunn, S., Putallaz, M., Sheppard, B. y Lindstrom, R. (1987). Social Support and Adjustment in Gifted Adolescents. Journal of Educational Psychology, 79(4), 467-473.

Escobar, M., Blanca, M.J., Fernández-Baena, F.J. y Trianes, M.V. (2011). Adaptación española de la escala de manifestaciones de estrés del Student Stress Inventory (SSI-SM). Psicothema, 23 (3), 475-485. 
Feldman, L., Goncalves, L., Chacón-Puignau, G., Zaragoza, J., Bagés, N. y Pablo, J. (2008). Relaciones entre estrés académico, apoyo social, salud mental y rendimiento académico en estudiantes universitarios venezolanos. Universitas Psychologica, 7(3),739-751.

Fernández, L., Siegrist, J., Rödel, A. y Hernández, R. (2003). El estrés laboral un nuevo factor de riesgo ¿qué sabemos y qué podemos hacer? Atención Primaria, 31 (18), 524-26.

Fernández-Seara, J.L. (1992). EAE: Escalas de Apreciación del Estrés: manual. Madrid: TEA Ediciones S.A.

Ferrando, P.J., Chico, E.y Tous, J.M. (2002). Propiedades psicométricas del test de optimismo Life Orientation Test (LOT). Psicothema, 14(3), 673-680.

Fimian, M.J., Fastenau, P.A., Tashner, J.H., y Cross, A.H. (1989). The measure of classroom stress and burnout among gifted and talented students. Psychology in the Schools, 26, 139-153. DOI: 10.1002/1520-6807(198904.

Goldsmith, R. (1986). Dimensionality of Rosenberg Self-esteem Scale. Journal of Social Behaviour and Personality, 1(2), 253-264.

Goleman, D. 1995). Emotional Intelligence. Nueva York: Bantam Books (Trad. Castellano, Barcelona: Kairós, 1996)

González-Fortaleza, y Ruiz, E. (1993). Autoestima y sintomatología depresiva en adolescentes mexicanos: un estudio correlacional por género. Revista $A B P-A P A L, 15$, (2), 59-64.

Holmes, T.H., y Rahe, R.H. (1967). The Social Readjustment Rating Scale. Journal of Psychosomatic Research, 11, 213-218.

Hernández, J.M., Pozo, C. y Polo, A. (1994). La ansiedad ante los exámenes. Un programa para su tratamiento de forma eficaz. Valencia: Promolibro.

Hombrados, M.I., Gómez, L., Domínguez,J.M., García, P. y Castro,M. (2012). Types of social support provided by parents, teachers and classmates during adolescence. Journal of Community Psychology, 40(6), 645-664. DOI: 10.1002/jcop.20523.

Hombrados, M.I., García, M.A.y Gómez, L. (2013). The relationship between social support, loneliness, and subjective well-being in a Spanish sample from a multidimensional perspective. Social Indicators Research, 114, 1013- 34. DOI: 10.1007/s11205-012-01875.

Kiecolt-Glaser, J.K., Glaser, R., Strian, E.C., Stout, J.C., Tarr, K.L., Holliday, J.E. y Speicher, C.E. (1986). Modulation of cellular immunity in medical students. Journal of Behavioral Medicine, 9, 5-21. 
Kang, S., Shaver, P., Sue, S., Min, K. y Jing, H. (2003). Culture-specific patterns in the prediction of life satisfaction: Roles of emotion, relationship quality, and self-esteem. Personality and Social Psychology Bulletin, 29, 1596-1608. DOI: $10.1177 / 0146167203255986$.

Lazarus, R.S., y Folkman, S. (1986). Estrés y procesos cognitivos. Barcelona: Martínez Roca. Londoño, C. (2009). Optimismo y salud positiva como predictores de la adaptación a la vida universitaria. Acta Colombiana de Psicología, 12(1), 95-107.

Maldonado, E., Trianes, M.V., Cortes, A., Moreno, E. y Escobar, M. (2009). Salivary Cortisol Response to a Psychosocial Stressor on Children Diagnosed with AttentionDeficit/Hyperactivity Disorder: Differences Between Diagnostic Subtypes. The Spanish Journal of Psychology, 12(2), 707-714. DOI: 10.1017/S1138741600002079g.

Manso, J.F. (2010). Análisis psicométrico de la escala de autoestima de Rosenberg en una muestra de estudiantes universitarios. Revista Electrónica de Trabajo Social [edición online], 8.

Martín, I.M. (2007). Estrés académico en estudiantes universitarios. Apuntes de Psicología, $25(1), 87-99$.

Martín, L., Núñez, J., Navarro, J., y Grijalvo, F. (2007). The Rosenberg Self-Esteem Scale: Translation and validation in university students. The Spanish Journal of Psychology, $10(2), 458-467$.

Martínez, A., Reyes del Paso, G.A., García, A., y González, M.I. (2006). Optimismo/pesimismo disposicional y estrategias de afrontamiento. Psicothema, 18 (1), 66-72.

Misra, R. y McKean, M. (2000). College'students academic stress and its relation to their anxiety, time management, and leisure satisfaction. American Journal of Health Studies, 16, 41-51.

Muñoz, F.J. (1999). El estrés académico: incidencia del sistema de enseñanza y función moduladora de las variables psicosociales en la salud, el bienestar y el rendimiento de los estudiantes universitarios. Tesis doctoral. Facultad de Psicología de la Universidad de Sevilla.

Muñoz, F.J. (2003). El estrés académico. Problemas y soluciones desde una perspectiva psicosocial. Huelva: Publicaciones Universidad de Huelva.

Murray, S.L., Griffin, D.W., Rose, P., Bellavia, G.M.(2003). Calibrating the sociometer: The relational contingencies of self-esteem. Journal of Personality and Social Psychology, 85, 63-84. DOI: 10.1037/0022-3514.85.1.63. 
Musitu, G. y Cava, M.J. (2003). El rol del apoyo social en el ajuste de los adolescentes. Intervención Psicosocial, 12(2), 179-192.

Navarro, E., Tomás, J.M. y Oliver, A. (2006). Factores personales, familiares y académicos en niños y adolescentes con baja autoestima. Boletín de Psicología, 88, 7-25.

Paradise, A. y Kernis, M. (2002). Self-esteem and psychological well-being: Implications of fragile self-esteem. Journal of Social and Clinical Psychology, 21(4), 345-361. DOI: 10.1521/jscp.21.4.345.22598.

Peterson, C., Seligman, M.E.P. y Vaillant, G.E. (1988). Pessimistic explanatory style is a risk factor for physical illness: A third-five year longitudinal study. Journal of Personality and Social Psychology, 55(1), 23-27.

Polo, A., Hernández, J.M. y Pozo, C. (1996). Evaluación del Estrés Académico en Estudiantes Universitarios. Revista Ansiedad y Estrés, 2, 159-172.

Reid, A. (2004). Gender and Sources of Subjective Well-Being. Sex Roles, 51 (11/12), 617629. DOI: 10.1007/s11199-004-0714-1.

Remor, R., Amorós, M. y Carboles, J. (2006). El optimismo y la experiencia de ira en relación con el Malestar físico. Anales de Psicología, 22(1), 37-44.

Román, C. y Hernández, R. (2005). Variables psicosociales y su relación con el desempeño académico de estudiantes de primer año de la Escuela Latinoamericana de Medicina [Versión online]. Revista Iberoamericana de Educación, 37(2), 1-8.

Rosenberg, M. (1965). Society and the adolescent self-image. Princeton: Princeton University Press.

Saarni, C. (1999). The development of emotional competence. Nueva York, Guild-ford Press. Scheier, M. y Carver, C.S. (1985). Optimism, Coping, and Health: Assessment and Implication of Generalized Outcome Expectancies. Health Psychology, 4(3), 219-247.

Scheier, M.F., Carver, C.S. y Bridges, M.W. (1994). Distinguishing Optimism from Neuroticism (and trait anxiety, self mastery and self esteem): A reevaluation of the Life Orientation Test. Journal of Personality and Social Psychology, 67(6), 1.0631.078. DOI: 10.1037/0022-3514.67.6.1063.

Seligman, M.E. (2003). La auténtica felicidad. Barcelona. Madrid España: Verg.

Selye, H. (1978). The stress of life. New York: McGraw Hill.

Shapiro, L.E. (1997). La inteligencia emocional en niños. Madrid: Javier Vergara. Schmitt, D., y Allik, J. (2005). Simultaneous administration of the Rosenberg Self-Esteem Scale in 53 nations: Exploring the universal and culture-specific features of global 
Relaciones entre estrés académico, apoyo social, optimismo-pesimismo y autoestima en estudiantes universitarios

self-Esteem. Journal of Personality and Social Psychology, 89(4), 623-642. DOI: 10.1037/0022-3514.89.4.623.

Viñas, F. y Caparrós, B. (2000). Afrontamiento del período de exámenes y sintomatología somática autoinformada en un grupo de estudiantes universitarios. Psicología.com, (edición online) 4(1), 37-48. 